\title{
Cooperación en la Red de Bibliotecas del Instituto Cervantes. Dos ejemplos de actuación
}

\author{
Por Irene Martín-Montalvo-Cortés, María-Victoria Valverde-Facal y Yolanda De-la-Iglesia-Sánchez
}

\begin{abstract}
Resumen: Se presentan las características de la Red de Bibliotecas del Instituto Cervantes relacionadas con la cooperación entre sus bibliotecas, y dos proyectos en fase de realización. Se comienza con una exposición sobre el marco de actuación de las 61 bibliotecas como red española internacional de bibliotecas especializadas. A continuación se sintetizan dos proyectos cooperativos que se van a implantar próximamente, una base de datos común para la gestión del conocimiento y un plan de gestión cooperativa de las publicaciones periódicas. Finalmente se reflexiona sobre el valor de la cooperación en las bibliotecas.

Palabras clave: Cooperación bibliotecaria, Redes de bibliotecas, Bibliotecas especializadas, Gestión del conocimiento, Publicaciones periódicas.
\end{abstract}

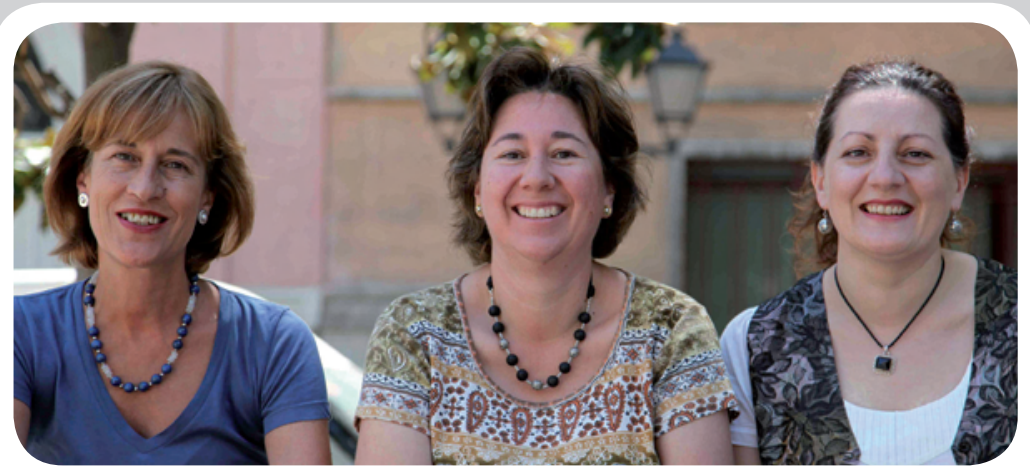

Irene Martín-Montalvo-Cortés es licenciada en psicología y jefa del Departamento de Bibliotecas y Documentación del Instituto Cervantes.

María-Victoria Valverde-Facal, licenciada en historia medieval y en documentación, es bibliotecaria en el Departamento de Bibliotecas y Documentación del Instituto Cervantes.

Yolanda De-la-Iglesia-Sánchez, licenciada en historia del arte y en documentación, es documentalista en el Depto. de Bibliotecas y Documentación del Instituto Cervantes, y profesora asociada en la Fac. de Documentación de la Univ. de Alcalá de Henares.

\section{Title: Cooperation at the Instituto Cervantes Library Network. Two case studies}

Abstract: The article presents the characteristics of the Instituto Cervantes Library Network in relation with the cooperation existing between them, as well as the two main projects under development. Firstly, there is an introduction to the frame of action of the 61 special libraries as an international network. The two aforementioned projects are explained in detail: a common database for knowledge management and a cooperative plan for periodicals administration. There is a final point considering the value of cooperation between libraries.

Keywords: Library cooperation, Libraries networks, Special libraries, Knowledge management, Periodicals administration.

Martín-Montalvo-Cortés, Irene; Valverde-Facal, María-Victoria; De-la-Iglesia-Sánchez, Yolanda. “Cooperación en la Red de Bibliotecas del Instituto Cervantes. Dos ejemplos de actuación”. El profesional de la información, 2010, septiembre-octubre, v. 19, n. 5, pp. 486-491.

DOI: $10.3145 /$ epi.2010.sep.06

\section{Introducción}

PRESENTES EN MÁS DE 42 PAÍSES, las 61 bibliotecas del Instituto Cervantes tienen como misión difundir la lengua española y las culturas de la comunidad hispanohablante. Mediante sus colecciones y servicios dan estabilidad a la política cultural de la institución y constituyen puntos de encuentro para fomentar el conocimiento, uso e investigación de la lengua española. Conforman la única red española internacional de bibliotecas especializadas.
Esta singularidad determina la organización y funcionamiento de la Red. De hecho, la dispersión geográfica de sus sedes, el ámbito multilingüe en el que se trabaja, los hábitos socioculturales de los diversos países en los que están ubicadas las bibliotecas junto a la disparidad en los niveles de desarrollo tecnológico alcanzado en cada uno de ellos, requieren una gran capacidad de adaptación a estas peculiaridades y a la constante evolución habitual en las tareas bibliotecarias. Por una parte están estrechamente ligadas a las tecnologías de la información y comunicación, y por otra tienen la obligación de dar respuesta a una demanda social múltiple y variable. Todos estos elementos han dado lugar a un modelo de gestión flexible, que permite aprovechar las oportunidades y disminuir el efecto de los puntos más débiles, en una tendencia a equilibrar la Red.

El Instituto Cervantes está formado por centros culturales y académicos repartidos por todo el mundo y una sede central desde la que se coordinan todas sus actividades. Esta estructura orgánica favorece 


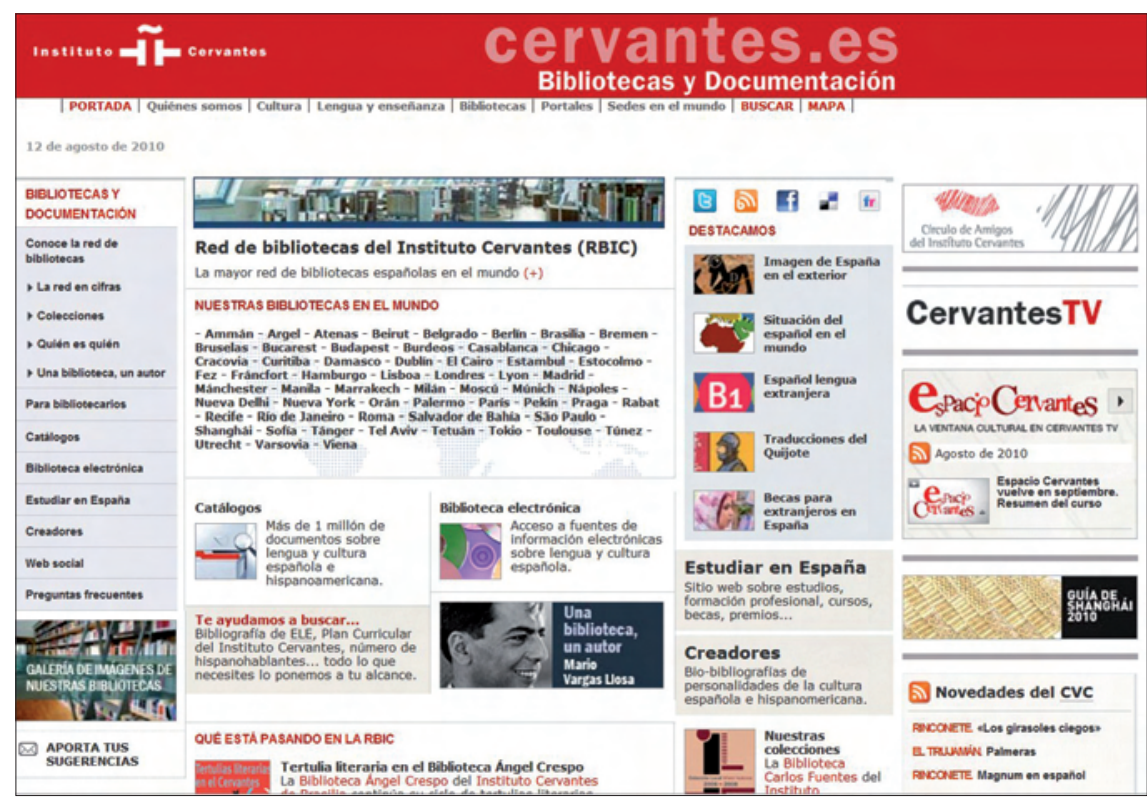

Portal de la Red de Bibliotecas del Instituto Cervantes

especialmente el trabajo en red, y los proyectos cooperativos. Desde el inicio de la implementación de la Red de Bibliotecas muchos planteamientos fueron concebidos sobre la base de la cooperación. Un buen ejemplo de ello son las políticas relativas a los fondos bibliográficos, gestionados en un único catálogo colectivo. Si bien la composición de las colecciones de las bibliotecas tiene un inevitable porcentaje de coincidencias, cada una lleva a cabo su propia selección bibliográfica, manteniendo "secciones especiales" sobre autores o materias que no se repiten sino que se complementan formando un conjunto con el resto de las bibliotecas.

La organización centralizada no impide una autonomía funcional en las relaciones bidireccionales entre las bibliotecas y los países anfitriones. Las bibliotecas favorecen el intercambio de conocimientos: en unos casos recogen buenas prácticas y recursos novedosos en el quehacer bibliotecario, y en otros aportan su experiencia en este campo profesional.

Aunque aún caben muchos avances, se ha ido tejiendo una red de relaciones cooperativas compleja, que se desenvuelve en diferentes niveles e implica a distintas institu- ciones. Existe un tipo de cooperación interna por áreas linguiísticas, por intereses comunes, etc. Asimismo, cada biblioteca suele asociarse -por separado o junto con otras bibliotecas del Instituto Cervantes en el mismo país-con instituciones locales para abordar proyectos conjuntos, pero además pueden colaborar con alguna institución española. La Red en su globalidad colabora con otras redes.

\section{"Misión: difundir la lengua española y las culturas de la comunidad hispanohablante"}

Las actividades cooperativas que se llevan a cabo son muy numerosas, variadas y están abiertas a cualquier novedad. No se trata aquí de teorizar sobre los beneficios de la cooperación, de sobra conocidos, sino de ofrecer un punto de vista práctico mediante la presentación de dos proyectos que se van a implantar próximamente en la $R B I C$, que mejorarán notablemente la gestión y supondrán un destacado incremento de los servicios a los usuarios.

\section{Dos proyectos en marcha}

\subsection{Base común de conocimiento}

Desde 1996 el centro de documentación de la sede central del Instituto ha prestado atención y soporte a las demandas de información y documentación tanto internas (originadas por el personal de la organización) como externas (público en general), así como las de los servicios de información de las bibliotecas de los centros (usuarios con carnet).

Para la administración de las preguntas y las respuestas se diseñó una matriz de operaciones sobre un sistema de gestión documental. El análisis y la valoración de las solicitudes de información ha contribuido a la planificación de los servicios y productos de información de la Red de Bibliotecas y del Departamento de Bibliotecas y Documentación.

El sistema documental compila las tareas, resultados y recursos necesarios para conducir todas las peticiones de información recibidas y sirve ahora de núcleo para un proyecto de gestión del conocimiento mediante una base de datos común.

\section{Gestión del conocimiento para exportarlo a la Red de Bibliotecas}

Unos de los principales productos de la gestión del conocimiento explícito son los almacenes o repositorios concebidos como bases de datos, pues ayudan a tomar las decisiones más acertadas en cada caso.

Conocer el contexto, el origen de la pregunta, las coordenadas del usuario que la realizó, el momento en que se efectuó, etc., son detalles que contribuirán a mejorar las respuestas sucesivas cuando se repiten las preguntas en parte, en su totalidad, o con variantes.

El proyecto cooperativo pasa por cinco grandes líneas de actuación: 
- Normalización

- Repositorio digital

- Fuentes de información de calidad

- Laboratorio de edición de contenidos web

- Evaluación y planificación de servicios y productos

a) Normalización. Los distintos métodos de edición y publicación de productos de información que hasta ahora han convivido en la Red pasarán a concretarse en un manual de estilo y en un manual de procedimiento. Ambos documentos van a recoger la imagen corporativa de cada biblioteca y de la Red en general para presentar a los usuarios una cara común, elaborada, con identidad. En la intranet Los bibliotecarios dispondrán de documentos con modelos de respuesta, pautas de análisis documental, o apariencia de los datos.

\section{"Manuales de estilo y de procedimiento recogerán la imagen corporativa de cada biblioteca y de la Red en general"}

La homogeneización abarca datos de diversa índole que conciernen a tres ámbitos:

- datos más representativos de cada consulta;

- documentos que la componen (físicos y virtuales), carta que envía el usuario, correos electrónicos de los usuarios y de las bibliotecas con la respuesta o con la solicitud a las fuentes institucionales, etc., además de cualquier dato representativo para el seguimiento y resolución de la consulta;

- documentos generados para la resolución de la consulta -dossier, bibliografía, biografía, listado, etc.- que son los genuinos productos de información.

b) Repositorio digital de productos de información. En el fondo documental fundacional se cuenta con miles de documentos generados desde 1994, monografías, informes técnicos, estudios, repertorios, biografías, bibliografías, etc. El trabajo conjunto va a consistir en ampliar y utilizar todos los productos elaborados por los bibliotecarios, así como los documentos normalizados y contemplados ahora en un depósito común disponible para el grupo. Desde hace tiempo se ha visto la repetición de dosieres informativos y otros frutos de la faena informativa entre las bibliotecas. Se han emprendido labores de unificación con algunos contenidos señalados como los referidos a personalidades de la cultura española e hispanoamericana, reflejo del apoyo de los servicios de información a las labores de gestión cultural de los centros del Instituto, organizadas en la web institucional en la sección Una biblioteca, un autor, y cuyo cometido es aglutinar toda la información disponible sobre los autores que dan su nombre a las bibliotecas de la Red.

El acopio de documentos se basa en el conocimiento de fuentes de información y en las peticiones específicas de los usuarios. Papel importante supone la recopilación de todos aquellos informes y documentos producidos con carácter interno y capaces de sugerir vínculos institucionales que permitan la colaboración con organismos de interés para la cultura y la lengua españolas y así poder encauzar otras potenciales demandas de información.

c) Fuentes de información de calidad y control de las fuentes especializadas. Para encontrar las respuestas a las preguntas a menudo es necesario recurrir a varias fuentes. La base de datos facilita tanto las fuentes que dieron re- sultados incompletos o negativos como las que propiciaron la resolución de la consulta. Este acopio de fuentes se acompaña de una evaluación simultánea de la calidad, teniendo en cuenta los criterios estándar de exactitud en los datos (coordenadas para localizar un autor, en distintas épocas y de todas las formas posibles, correo electrónico, o dirección postal); autoría de las fuentes, importa mucho el quién es quién que notifica los datos; objetividad, análisis de cada fuente y sus fines y utilidades para según qué propósitos u objetivos de búsqueda; actualidad, ¿cuándo se realizó?, ¿cuándo se actualizó?; y cobertura, que viene a ser en este caso hasta dónde llega la fuente en sus acepciones y sus posibilidades de reutilización.

\section{"Se van a añadir al repositorio todos los productos concebidos por los bibliotecarios, así como los documentos normalizados"}

La base datos va a proporcionar grandes beneficios al convertirse en un recurso propio y exclusivo, de referencia y fuente, es decir, para obtener las respuestas concretas o para saber dónde encontrarlas. Tiene gran interés la recopilación de datos con nombres propios de entidades, lugares y personas, que de otra manera no son fáciles de localizar. La información local de cada país o entorno cultural es otra de las aportaciones más considerables que las bibliotecas van a poner en práctica.

d) Laboratorio de edición de contenidos destinados a la web pública (portal de las bibliotecas), a las redes sociales (Facebook, Twitter y distintos blogs) o la web 


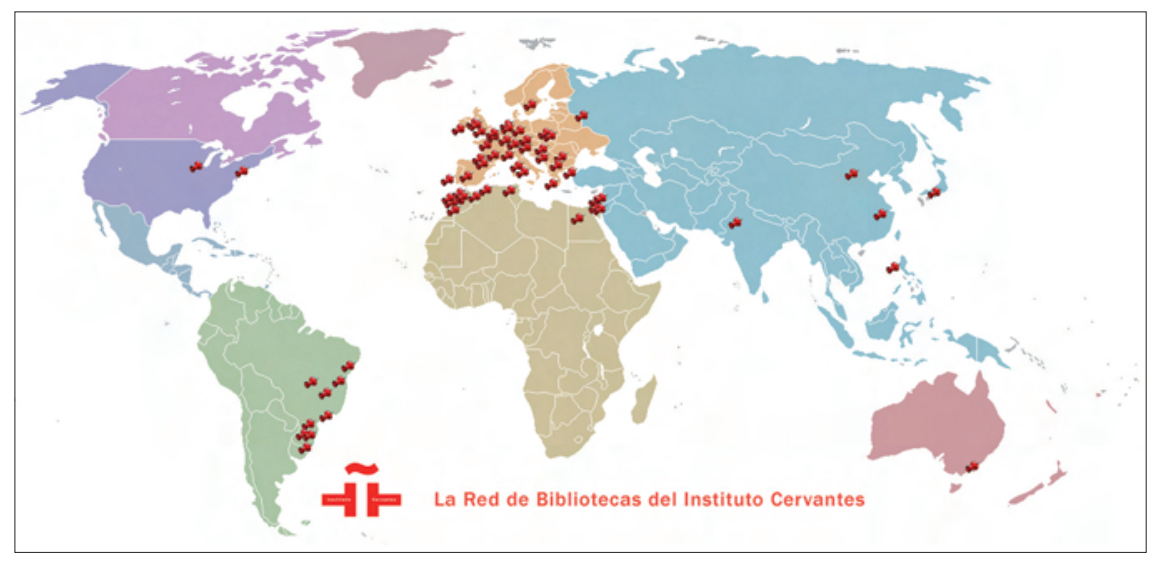

Presencia del Instituto Cervantes en el mundo

corporativa (intranet). Los productos elaborados para la resolución de las consultas tras su almacenamiento se filtran para amortizar su uso y explotación: unos son objeto de permanente actualización, otros tienen un ciclo vital efímero, otros se actualizan bajo demanda y otros pueden constituir un contenido de calidad, que puede alojarse en la web, bien la pública o social (selección de documentos como dosieres temáticos electrónicos, convocatoria y seguimiento de eventos) o bien la corporativa (informes internos).

El esfuerzo cooperativo se centra en la producción de contenidos de interés para los usuarios por parte de los bibliotecarios, por necesidades de información de tipo general, o por grupos de interés. La contribución de las bibliotecas para gestar contenidos comunes descansa sobre la herramienta informática de edición de páginas web de la institución, y va desde la traducción en equipo de información sobre características y uso de algunos servicios de información, a la alimentación de secciones completas en el portal como la de Qué está pasando en la RBIC, desde donde se difunden diariamente eventos, actividades o propuestas de las bibliotecas.

La colaboración en las redes sociales cobra cada vez más fuerza. Los bibliotecarios comparten sus relaciones con los usuarios interesados en la lengua y la cultura en español desde varias redes sociales, con páginas comunes o con sindicación de contenidos. Se puede mantener con efectividad y eficacia una comunicación interactiva con los usuarios. Hay propuestas de consolidación del papel de las bibliotecas de la Red en la web 2.0 con nuevas iniciativas como clubs de lectura virtuales.

\section{"Los bibliotecarios comparten sus relaciones con los usuarios interesados en la lengua y la cultura en español desde varias redes sociales"}

e) Evaluación y planificación de servicios y productos. El control de calidad de los servicios de información pasará a ser una realidad cuando con la participación de las bibliotecas en la elaboración y el análisis de los datos se alcancen mayores cuotas de evaluación, tanto interna como externa. Datos cuantitativos y cualitativos -tipo de usuarios, tiempos de respuesta, fuentes de información a las que recurre con mayor frecuencia, formas más comunes de recepción de la consulta, etc.- que en la actualidad no se recogen sistemáticamente por carecer de instrumentos específicos, y de la metodología pertinente. Has- ta ahora el estudio de los servicios de información en las bibliotecas de la Red ha sido muy superficial y con métodos manuales. A partir de la implantación de la gestión del conocimiento la recogida de datos será constante, así como la articulación de documentos de mejora que faciliten la planificación de nuevos y mejores servicios, contenidos $y$, en definitiva, formas de comunicación con los usuarios.

\section{Conclusiones}

Se trata de llegar a un modelo que administre conocimiento y no mera información, que evolucione de una gestión centralizada a una gestión cooperativa, facilitada por un cambio en la aplicación informática para la gestión del conocimiento. Las 61 bibliotecas pasarán a alimentar el repositorio de documentos y de procedimientos y serán artífices de una serie de hechos, algunos de las cuales ya se pueden prever:

- incremento de fuentes locales e internacionales;

- futuro sistema de referencia digital;

- ahorro de tiempo;

- mejor autoridad intelectual (calidad de las fuentes);

- actualización mayor y más dinámica de los contenidos;

- lanzamiento de iniciativas de nuevos productos y servicios;

- indicadores específicos para el control de calidad;

- innovación en la gestión del conocimiento;

- cooperación con otras bibliotecas o redes bibliotecarias.

\subsection{Gestión cooperativa de las publicaciones periódicas}

\section{Situación actual}

La colección de revistas locales de la $R B I C$ se puede agrupar en:

- Hispanismo y didáctica del español. 
- Cultura española e hispanoamericana.

Ambos temas responden a la materia de especialización de nuestras bibliotecas, pero existen algunas diferencias entre ellos: si bien las revistas locales se reciben por suscripción y sólo están en las bibliotecas del país que las edita, las revistas españolas están presentes en todas las bibliotecas y se reciben mediante donación. Son enviadas desde España a cada biblioteca pero, por motivos burocráticos, de infraestructura y de dificultad en su envío, no están regularmente distribuidas; las colecciones a veces están incompletas o tienen duplicados.

La colección de publicaciones periódicas se gestiona mediante el catálogo colectivo. Para cada título se describen los fondos y se detallan los números de revista que cada biblioteca tiene. Por otro lado, la revista también está dada de alta en la biblioteca donde se encuentra. Las mencionadas dificultades en la recepción de las revistas restan calidad al servicio, puesto que su principal interés es la actualidad de su información.

Existe un problema técnico en la presentación de la información en el opac. Muchas bibliotecas prestan los fascículos de las revistas, para lo cual los dan de alta en el programa informático. Esto ocasiona que, para aquellas revistas con muchos números al año y que están presentes en la mayoría de las bibliotecas, la presentación de resultados en el opac se convierta en una lista interminable de ítems que en ocasiones el sistema no es capaz de mostrar en su totalidad.

El almacenamiento de la colección también necesita una revisión, pues si bien el uso de las revistas durante el año en curso y el siguiente es intensivo, a partir del segundo año se reduce, pasando a ser sólo fuente de información para eventuales investigaciones. Esto requie- re diseñar un sistema de suministro de información y conservación adecuado a esta nueva finalidad.

\section{"El plan de gestión cooperativa de publicaciones periódicas pretende liberar espacio y hacer más clara la información sobre los fondos existentes"}

\section{Actuaciones previstas}

Para ganar eficacia y mejorar los servicios, se ha elaborado un plan de gestión cooperativa de las publicaciones periódicas entendiendo que se trata de una única colección cuya ubicación y gestión está repartida entre todas las bibliotecas de la Red.

Algunas bibliotecas ya tienen compartidas las suscripciones de revistas, de manera que las bibliotecas de un mismo país se ponen de acuerdo y se reparten las suscripciones evitando así la duplicidad de colecciones. A partir de la puesta en marcha de este plan, esta fórmula para la suscripción se va a hacer extensiva a toda la Red en aquellas áreas geográficas en las que se pueda.

Ya se ha explicado antes cómo se realiza en este momento el control bibliográfico en el programa informático y su problemática.

Este plan de gestión cooperativa plantea un cambio importante en lo relativo a los préstamos ya que se podrán realizar mediante la asignación de un número fijo de códigos de barras a cada título, hasta un máximo de cinco.

De esta manera se consigue que la visualización en el opac se acorte, facilitando la consulta de las existencias de cada biblioteca.
Cada ficha contendrá la siguiente información: título de la revista, códigos de barras de la revista y un espacio para anotar el número del fascículo prestado. El préstamo se realizará de manera automatizada en el programa informático, como un préstamo más, leyendo con el lector óptico el código de barras pegado en la ficha en papel y el código de barras del lector. En la ficha en papel se anotará el número del fascículo que se ha prestado, y se borrará en el momento en que sea devuelto, de manera que el código de barras pueda ser reutilizado en el siguiente préstamo.

De las revistas editadas en España sobre temas culturales, cada biblioteca mantendrá el año en curso y el anterior, retirando o expurgando todos los años anteriores. Para garantizar la conservación de las revistas más interesantes, cada biblioteca se comprometerá a mantener tres títulos determinados actualizados y lo más completos posible.

Las nuevas condiciones de préstamo se establecerán en un documento normativo que deberán seguir todas las bibliotecas de la $R e d$, y se basará en unos principios racionales teniendo en cuenta el modo en el que a partir de ahora se va a gestionar toda la colección de revistas. Las revistas anteriores a dos años no podrán ser prestadas a domicilio por la biblioteca que las conserva, pero ofrecerá el servicio de suministro de artículos a todas las bibliotecas de la Red y a cualquier usuario que lo solicite. La biblioteca que recibe la petición digitalizará el artículo, lo suministrará al usuario por la vía que se precise, y guardará una copia del documento digitalizado en un repositorio común, de manera que si ese mismo artículo es solicitado por otro usuario en otra biblioteca, cualquier bibliotecario de la Red pueda obtenerlo sin necesidad de volver a digitalizarlo. 
Para el caso de las revistas del año en curso y del anterior, la biblioteca seguirá ofreciendo sus servicios como habitualmente ha hecho pero utilizando, para el préstamo, la ficha en papel arriba mencionada.

\section{Conclusiones}

El objetivo que se persigue con la puesta en marcha de este plan de gestión cooperativa de publicaciones periódicas es múltiple: liberar espacio, hacer más clara la información sobre los fondos existentes y mejorar la eficacia de los servicios.

La Red de Bibliotecas del Instituto Cervantes tiene aproximadamente 31.357 metros lineales de estantería, y en ellos debe encajar 1.129.981 volúmenes que conforman su colección bibliográfica. La posibilidad de liberar espacio es uno de los motivos que lleva a diseñar una serie de actuaciones para gestionar la colección de revistas de otro modo. Racionalizando el espacio se espera ganar una media de 44 metros de estantería por biblioteca.

La simplificación de la información en el opac facilitará su comprensión y permitirá que el usuario obtenga la información completa. La notación de fondos ofrecerá la información de los números de revista que se tienen, y el alta de ejemplares cumplirá su función de permitir el préstamo automatizado.

Finalmente, mejorará la eficacia y rapidez de los servicios de docu- mentación por la facilidad que supone acudir al repositorio en busca de los artículos digitalizados de las revistas que no se tengan.

\section{Consideraciones finales}

Los servicios de información del siglo XXI no necesitan ya apoyarse tanto en el espacio y la presencia física. En un mundo interconectado y con información virtual de calidad, se hacen posibles nuevas fórmulas de organización de los servicios que aprovechan mejor los recursos compartiendo las cargas de trabajo. Sin embargo, a día de hoy todavía no se puede prescindir de las estanterías donde conservar publicaciones que aún no tienen su versión digital, por lo que el espacio físico se revaloriza obligando a racionalizar su uso.

Se han seleccionado dos proyectos por encontrarse en la fase previa a su implantación y porque son las propuestas de cooperación más recientes en la RBIC. Además son dos ejemplos que apuestan por la aplicación de fórmulas cooperativas tanto en el entorno físico como en el virtual. La mejora continua en la atención a los usuarios y la racionalización de los recursos obligan a plantear propuestas versátiles y a diseñar nuevas alternativas para poder responder con las mayores cotas de calidad en los servicios a los usuarios.

En las redes de bibliotecas los servicios compiten con otras opciones en el mercado y sólo cuando se difunden tienen una oportunidad de ser utilizados. Por lo tanto, deben destacarse de alguna manera para sobresalir ante la cantidad de ofertas disponibles.

\section{Bibliografía}

Caffarel, Carmen. "[Bibliotecas del] Instituto Cervantes". En: Anuario de bibliotecas españolas 2009. Málaga: Fundación Alonso Quijano, 2009, pp. 254-259.

http://www.cervantes.es/imagenes/file/biblio teca/caffarel_2008.pdf

Gonzalo-Balmisa, Yolanda. "De Beirut a Pekín: experiencias informativas en la red de bibliotecas del Instituto Cervantes". En IX Jornadas de gestión de la información, 2007, pp. 93-102.

http://eprints.rclis.org/archive/00012253/01/ SedicJGI07-Gonzalo.pdf

Kempf, Klaus. "Unidos somos más fuertes Cooperación en las bibliotecas científicas alemanas". El profesional de la información, 2008 mayo-junio, v. 17 , n. 3, pp. 324-330

Martín-Montalvo, Irene. "La Red de Bibliotecas del Instituto Cervantes: origen y evolución". Métodos de información, 2002, enero-marzo, v. 9, n. 49, pp. 32-38.

http://www.cervantes.es/imagenes/file/biblio teca/rbic_irene.pdf

Valverde-Facal, María-Victoria; De-la-Iglesia-Sánchez, Yolanda. "La intranet como herramienta de gestión en la Red de Bibliotecas del Instituto Cervantes". Métodos de información 2002, enero-marzo, v. 9, n. 49, pp. 45-54.

http://www.cervantes.es/imagenes/file/biblio teca/mava_yolandai_intranet.pdf

Irene Martín-Montalvo-Cortes, María-Victoria Valverde-Facal, Yolanda De-la-Iglesia-Sánchez.

Departamento de Bibliotecas y Documentación. Dirección de Cultura, Instituto Cervantes.

Alcalá, 49 - $2^{a}$ planta. 28014 Madrid.

http://www.bibliotecas.cervantes.es irene@cervantes.es mava@cervantes.es yolandai@cervantes.es

\section{Suscripción EPI sólo online}

Pensando sobre todo en los posibles suscriptores latinoamericanos, ya no es obligatorio pagar la suscripción impresa de EPI para acceder a la online.

EPI se ofrece a instituciones en suscripción "sólo online" a un precio considerablemente más reducido (90 euros/año), puesto que en esta modalidad no hay que cubrir los gastos de imprenta ni de correo postal. 